\title{
A Comparative Study on Correlation between Personal Background and Interior Color Preference
}

\author{
Mahshid BANIANI $^{1}$, and Sari YAMAMOTO ${ }^{2}$ \\ ${ }^{1}$ Art and Design Department, Graduate School of Comprehensive Human Sciences, \\ University of Tsukuba \\ ${ }^{2}$ Faculty of Art and Design, University of Tsukuba
}

\begin{abstract}
In this research, we clearly identify and investigate some of the factors influencing residential color preference. We have taken social environment as a primary focus and the influence of a person's residential, regional, educational and personal background have been examined. A questionnaire, 2 drawings (one of a bedroom and another of the exterior of a few houses) and 24 color pencils were prepared. In total, 319 data were gathered from Japanese students living in Japan, Foreign students living in Japan and Iranian students living in Iran, with 301 applicable data. One of the questions was the respondent's favorite color. The results showed that more than $96.7 \%$ have used their favorite colors in the drawings. Blue hues were used most by people living in coastal areas $(P<0.05)$. Education had a strong correlation with number of color varieties $(P<0.01)$. Moreover, students who had parents with art backgrounds had used more colors in the drawings rather than the ones with parents with no art background $(P<0.05)$. Foreigners and Iranians have used more color varieties compared to Japanese $(P<0.05)$. Ultimately, it was observed that among the case studies (regional, residential, educational), education had a strong positive correlation with number of color varieties $(P<0.01)$.
\end{abstract}

Keywords: Color Varieties, Education, Environment, Color Preference, Personal Background, Regional, Drawings, Interior, Exterior

\section{Introduction}

In a previous research ${ }^{1}$, we did a cross cultural study regarding interior color preference and concluded that there is not much color variety when Japanese people are choosing colors for their bedroom walls and they are all focused on white more so than others. Furthermore, M. Saito has done a cross cultural study ${ }^{2}$, and concluded that Japanese people like white (more so than other respondents). In addition, Gunnerod $^{3}$ concluded that Japanese consumers prefer white, whereas consumers from Hong Kong prefer red. T. Kanda ${ }^{4}$ conducted a cross cultural study among Japanese kindergarten pupils, Japanese university students and foreign university students from Asia and concluded that orange is the most favorable color for candies and black is the most unfavorable one. Moreover, he concluded that preference of Japanese kindergarten pupils and Japanese university students are similar but differ from that of foreign university students from Asia on the whole. As known, there are several other studies regarding color preference across different cultures. For instance, Garth ${ }^{5}$ studied color preference using Indians, Caucasian, and the mixed-race of the two ethnic groups. Jacobs and colleagues $(1991)^{6}$ conducted a cross 
cultural study with student subjects from Japan, People's Republic of China, South Korea and United States. Silver ${ }^{7}$ reports that African Americans like colors in red-purple-black range, whereas white subjects prefer blues and greens. Similarly, Wiegersma and Elst ${ }^{8}$ report that blue is the color chosen most often, except by respondents from Senegal and Transkei, who prefer red and black. Shoyama et al. ${ }^{9}$ studied color preference for clothing using Japanese and Korean women as the observers. The results showed that the Japanese observers preferred black and light grayish orange, whereas Korean observers preferred dark blue and light gray.

As it can be seen all of these studies are focused on cultural influences and although, it can be concluded that culture plays an important role on color preference, but these studies don't show us if there are other factors influencing color preference.

Similarly, there are numerous studies regarding color preference and gender. Hurlbert and Ling ${ }^{10}$ found female observers preferred redder colors more than the male. Guilford and Smith ${ }^{11}$ concluded that men are more tolerant of achromatic colors compared to women. Garth and Porter ${ }^{12}$ examined color preference of 1032 young children and concluded that red was favored most by the boys and blue by the girls with red running a close second. Walton et al. ${ }^{13}$ reported that the university student male subjects preferred orange to yellow but for the female subjects the preference is reserved. Harris concluded that girls tend to prefer lighter colors than boys ${ }^{14}$.
These are just a few examples of studies regarding color preference and gender, however, these (and other) studies have been focused on the colors used or favored by each gender and although, preferences can clearly be observed across genders, however these studies do not cover other areas such as number of color varieties used in a drawing by each gender or etcetera. And they are mainly just focused on color preferences according to gender.

Moreover, there are also studies concerning color preference and age. Walsh et al. (1990) ${ }^{15}$ found that 5-year-old-children generally prefer red candy over green, orange and yellow candy. Read and Upington ${ }^{16}$ concluded that young children prefer red in interior environment. Zemach and Teller ${ }^{17}$ concluded that 12-week-old-infants showed preferences for color visual stimuli over white visual stimuli. Terwogt and Hoeslma ${ }^{18}$ reported that children disliked white and black. Similarly, Pitchford and Mullen (2005) revealed that children preferred gray and brown less than other basic colors ${ }^{19}$. Cimbalo et al. ${ }^{20}$ tested the association of color and emotion with primary and college students. Adams and Osgood ${ }^{21}$ studied color preference of high school students in 20 countries. Irvin et al. ${ }^{22}$ did an experiment with children in grades 1-12 using color pairs. Jadva et al. ${ }^{23}$ concluded that both boy and girl infants prefer reddish colors to blue colors, and rounded shapes to angular shapes.

However, all of these studies have mainly been focused only on gender differences across ages and they have not concluded if other factors influence color preference. Moreover, 
they are mainly focused on younger ages.

There are many other studies regarding color preference and culture, age and gender, however, these studies have never been taken further. Most people have relatively strong and idiosyncratic color preferences, but little is known why they have the preferences they do (Eysenck, 1941 ${ }^{24}$; Granger, 1955 ${ }^{25}$; Guilford \& Smith, 1959 ${ }^{11}$; McManus, Jones \& Cottrell, $1981^{26}$; Hurlbert \& Ling, $2007^{10}$ ). Neperud and Freeman $^{27}$ concluded that preference might be "personal idiosyncratic phenomena not tied to development considerations" (p.86). Perhaps, this phenomenon as they refer to it, is "an individual difference determined in part by learning experiences, past experiences, socialization, cultural values, and maturation" (Newton, 1989, p.77) ${ }^{28}$.

Therefore, in this research, we will take these studies further and through comparing different social and architectural contexts and experiments, we clearly identify and investigate some of the factors influencing color preference. We have taken social environment as our primary focus and the influences of a person's residential, regional, educational and personal backgrounds have been examined.

\section{Methodology}

\section{2-1. Respondents}

This was done between August 2011 and January 2012 in Iran and Japan among university students within the ages of 20 to 30 (Table 1).

The foreign respondents were from $\operatorname{Laos}(2$ subjects), Vietnam(15), China(8), Thailand(3), Cambodia(7), The Philippines(3), Korea(3), Tajikistan (2), Kazakhstan(2), Kyrgyzstan(7), Latvia(2), Estonia(2), Romania(2), Italy(3), Netherlands(3), France(2), Germany(4), USA (12), Brazil(1), Costa Rica(4), Nicaragua(1), Tunisia(1), and Malawi(3).

\section{2-2. Procedure and Method}

In the beginning, each respondent was given 2 drawings (Figure 1) and 24 color pencils and they were asked to paint the drawings according to their preferences. Although the word "interior" is used for the description of Figure 1, but we have picked a bedroom drawing and the reason for this is because bedroom is considered as someone's private room and it is one place where anyone can make the desired changes.
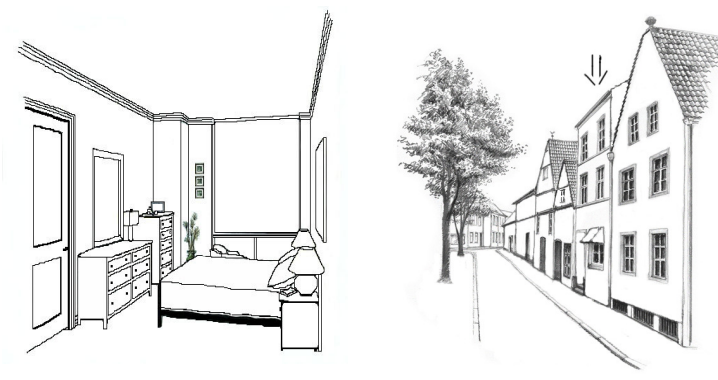

Figure 1. Sample of interior and exterior drawings

Table 1. Number of Gathered Data (Applicable Data)

\begin{tabular}{rrrr} 
Iranians Living in Iran & Japanese & Foreigners Living in Japan & Total \\
\hline $101(94)$ & $122(115)$ & $96(92)$ & $319(301)$ \\
$\mathrm{F}=47, \quad \mathrm{M}=47$ & $\mathrm{~F}=86, \mathrm{M}=29$ & $\mathrm{~F}=52, \mathrm{M}=40$ & $\mathrm{~F}=185, \mathrm{M}=116$ \\
& $F=$ Female, $M=$ Male &
\end{tabular}


After the drawings were done, they were handed a questionnaire regarding their personal background which was divided into 3 sections of regional, educational and residential. There were also a few personal questions regarding their major, nationality, favorite color, and background of their parents.

\section{Data Analysis and Results}

\section{3-1. Data Analysis}

The data was analyzed by comparing the regional, educational, residential and personal sections in the questionnaire and the number of the color varieties and the colors used in the drawings. Not only the number of color varieties and the colors used were considered, but also we verified if they have used any patterns on the walls, thus, to see the creativity, this method - drawings with the color pencils was the most appropriate.

In addition, in the questionnaire, there were questions (e.g. what is your favorite color) where the respondents were free to write any color they wanted. In these cases, we purposely matched the colors written by the subjects with the colors of the 24 color pencils (provided in the experiment) in order to do the analysis. Therefore, we narrowed down the colors written by the subjects to 24 .

Moreover, this paper mainly focuses on the analysis of the interior (bedroom) drawing, however for the sections that discuss both interior and exterior drawings, the exterior drawing's analysis only focuses on the façade and not the whole drawing since the main focus was the architecture and not the scenery.

\section{3-2. Usage of Favorite Colors in the Drawings}

One of the questions was the respondents' favorite color. The respondents were free to write any color they wanted, but as stated earlier, those color names were matched with the colors of the 24 color pencils. It was observed that all of the Iranians, $97.35 \%$ of the Japanese and $96.77 \%$ of the foreigners have used their favorite colors in the drawings (Table 2). One Japanese subject didn't use her favorite color (ultramarine blue) for the reason of not being suitable for architecture (interior or exterior). 3 foreigners didn't use their favorite colors: Gray (because the subject couldn't find the gray she was looking for in the color pencils, Black (not suitable) and Blue (her favorite color for clothes and not for architecture). In all the regions, the favorite colors were most used in the exterior drawing with foreigners having the highest percentage $(90.11 \%)$. Following that, the favorite colors were used most in the

Table 2. Distribution of favorite colors in the drawings

\begin{tabular}{|c|c|c|c|c|c|c|c|}
\hline & \multirow{2}{*}{ Same } & \multicolumn{5}{|c|}{ Interior (Bedroom) } & \multirow{2}{*}{$\begin{array}{r}\text { Exterior } \\
\text { Walls }\end{array}$} \\
\hline & & Wall & Bed & Door & Furniture & Smaller Objects & \\
\hline Iran $(N=94)$ & $100.0 \%$ & $56.4 \%$ & $38.3 \%$ & $18.1 \%$ & $16.0 \%$ & $76.6 \%$ & $76.6 \%$ \\
\hline Japan (N=115) & $97.4 \%$ & $20.4 \%$ & $31.9 \%$ & $21.8 \%$ & $22.7 \%$ & $77.9 \%$ & $88.5 \%$ \\
\hline $\begin{array}{l}\text { Foreigners } \\
(\mathbf{N}=92)\end{array}$ & $96.8 \%$ & $58.2 \%$ & $35.2 \%$ & $12.1 \%$ & $20.9 \%$ & $78.0 \%$ & $90.1 \%$ \\
\hline
\end{tabular}


smaller objects in the interior drawing (such as table lamp, picture frames and flower vase). More than half of Iranian and foreign subjects, have used their favorite color in the bedroom walls, whereas Japanese respondents only used $20.4 \%$ of their favorite colors in the bedroom walls.

\section{3-3. Regional and Color Varieties}

The respondents were asked to select the type of the place (in the city/town) they live in; being it Metropolitan, Coastal, Mountainous, or Others. As for the foreign subjects living in Japan, they were supposed to refer to the place(s) they have lived in before coming to Japan. The distribution for each region can be seen in Table 3. FR stands for foreigners.

Table 3. Distribution of city types Metropolitan Coastal Mountainous Others

\begin{tabular}{lllll}
\hline Iran & $55.3 \%$ & $0.0 \%$ & $44.7 \%$ & $0.0 \%$ \\
$(\mathrm{~N}=94)$ & & & & \\
\hline Japan & $38.3 \%$ & $21.7 \%$ & $33.9 \%$ & $6.1 \%$ \\
$(\mathrm{~N}=115)$ & & & & \\
\hline FR & $52.2 \%$ & $35.9 \%$ & $12.0 \%$ & $0.0 \%$ \\
$(\mathrm{~N}=92)$ & & & &
\end{tabular}

Figure 2 and Figure 3 show the number of color varieties used in the interior and exterior drawings in each region. In Japan, the subjects have used more number of color varieties in the exterior drawing compared to the interior drawing $(\mathrm{p}<0.05)$, although, this wasn't seen for Iranian and foreign respondents. In Iran, people living in metropolitan and mountainous areas have the same average of number of color varieties used in the interior drawing, while this average falls to 8.21 in the exterior drawing for people living in mountainous areas. In other words, people living in Iranian mountainous areas have used less number of color varieties in the exterior drawing compared to the interior. For Japanese and foreign respondents, people living in coastal areas had the lowest average of number of color varieties compared to the respondents living in other areas $(\mathrm{p}<0.05)$.
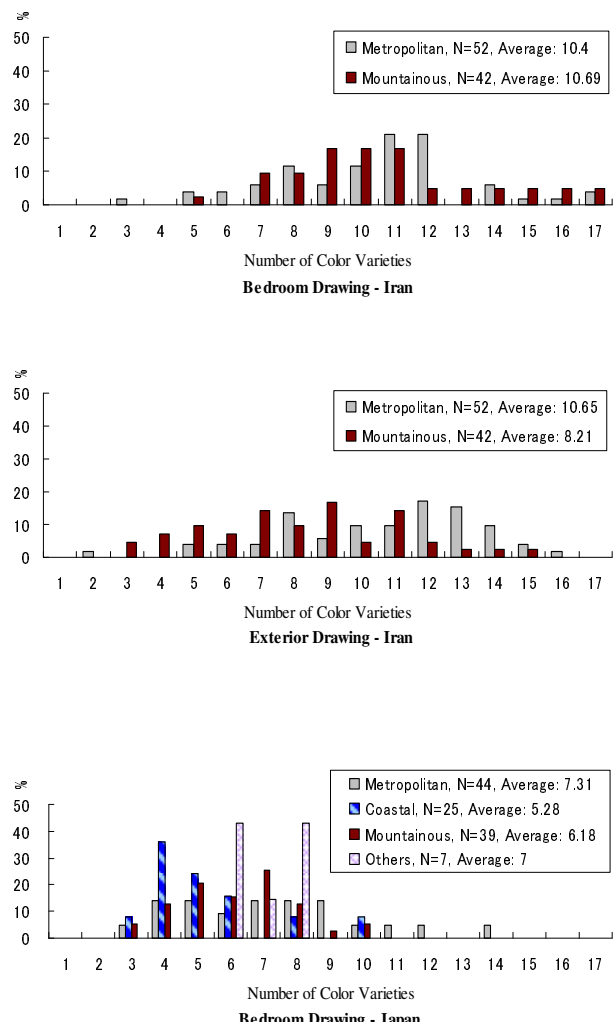
Bedroom Drawing - Japan

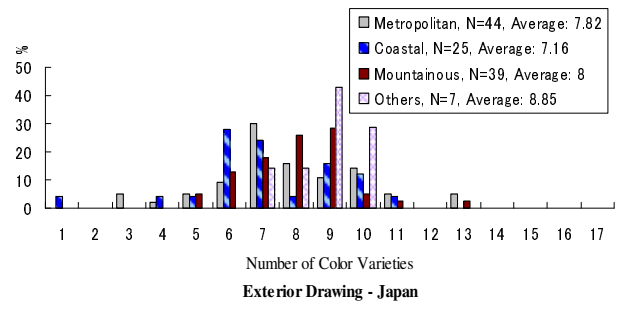

Figure 2. Number of color varieties in each region - Iranians and Japanese 

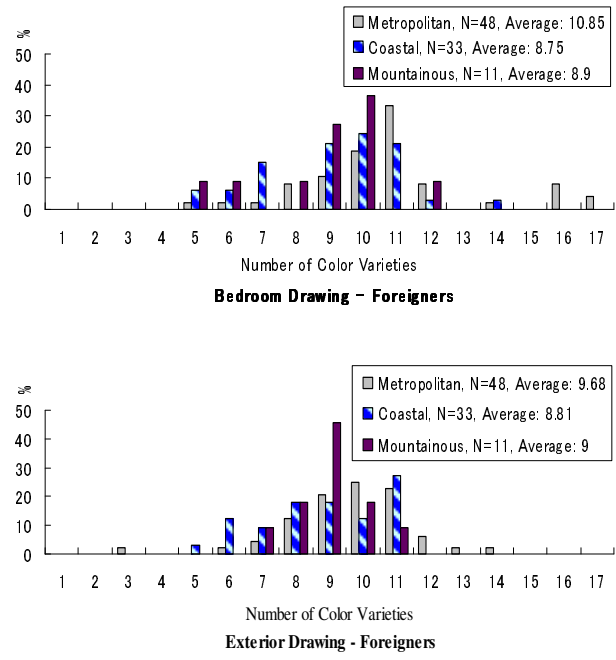

Figure 3. Number of color varieties in each region-Foreigners

\section{3-4. Residential and Color Varieties}

The Purpose of this section was to see if there is any correlation between the colors of the house(s) the subjects have lived in with the colors they used in the drawings or not. They were asked for the colors of their bedroom and exterior walls. In case they have lived in multiple houses, they were to give the colors for all the houses. Those answers were later compared with the drawings. The results can be seen in Table 4.

Table 4. Correlation between the subjects' own house and drawings

Exterior

Bedroom

\begin{tabular}{lrrrr} 
& Same & Different & Same & Different \\
\hline Iran (N=94) & $51.4 \%$ & $48.6 \%$ & $48.6 \%$ & $51.4 \%$ \\
\hline Japan (N=115) & $39.8 \%$ & $60.2 \%$ & $63.6 \%$ & $36.4 \%$ \\
\hline FR (N=92) & $56.9 \%$ & $43.1 \%$ & $52.6 \%$ & $47.4 \%$
\end{tabular}

As it can be seen from Table 4, Japanese subjects were more influenced by their bedroom wall colors rather than Iranian or foreigner subjects. Moreover, Japanese subjects were less influenced by their exterior wall colors. There wasn't a big difference between the Iranian and the foreign respondents regarding the influence of their own house.

\section{3-5. Education and Number of Color Varieties}

The respondents were asked to give the approximate hours of art classes they have had per week from kindergarten throughout high school. Figure 4 shows the number of color varieties that have been used in the bedroom drawing in each category according to the number of hours of art throughout their education (from kindergarten to high school), whereas Figure 5 focuses on the data as a whole (Iranian, Japanese and foreign subjects).
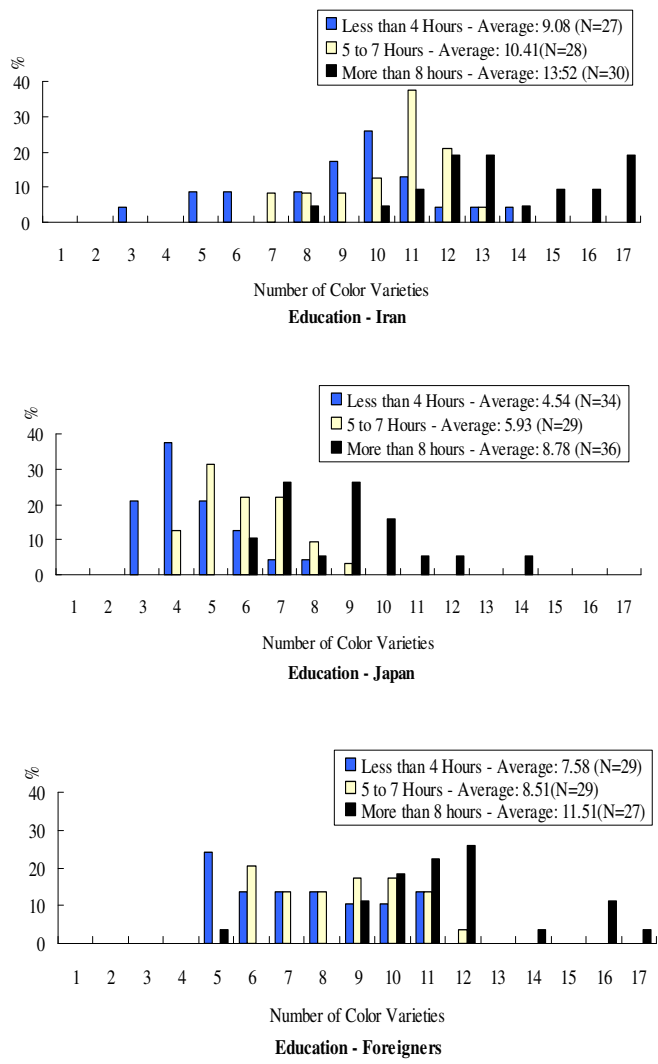

Figure 4. Number of color varieties and art-class hours through school (kindergarten to high school) 


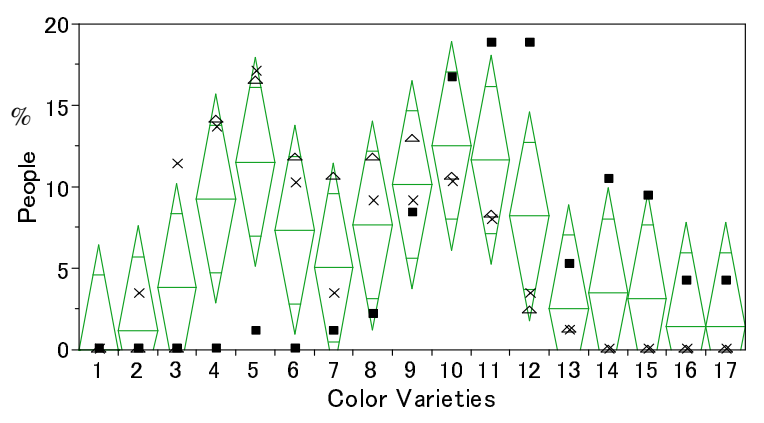

$\mathrm{X}$ : Less than 4 hours of art $\triangle 5$ to 7 hours

More than 8 hours of art

Figure 5. Education and number of color varieties

By looking at Figure 4 and 5, it is observed that with more hours of art classes, more number of color varieties are used in the bedroom drawing in all the categories $(\mathrm{P}<0.01)$.

Results from section "3-5" (Education and Number of Color Varieties) to section "3-7" (Respondents' Parents' Backgrounds and Number of Color Varieties) will only focus on the number of color varieties in the interior (bedroom) drawing. The same results were seen in the exterior drawing; therefore we don't see the need to include those results as well.

\section{3-6. Major and Number of Color Varieties}

As indicated earlier, there were a few personal questions; one of them being the respondents' major. Figure 6 shows the number of color varieties (in interior drawing) based on major in each category while Figure 7 sums up the data as a whole. It was concluded that in all the regions, the subjects who were art and design majored used more number of color varieties compared to those who weren't $(\mathrm{P}<0.05)$.
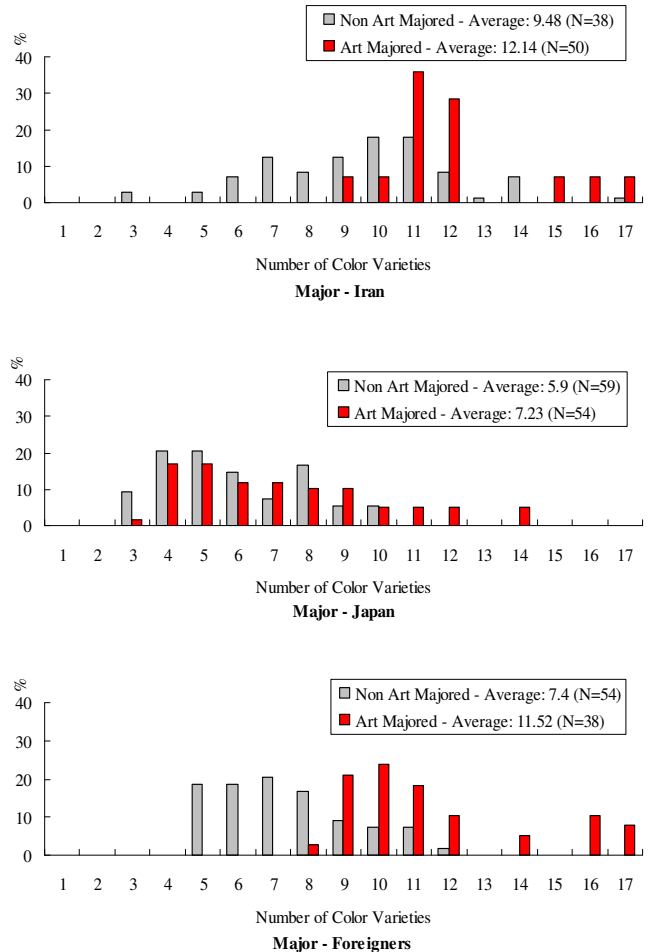

Figure 6. Major and number of color varieties in each category

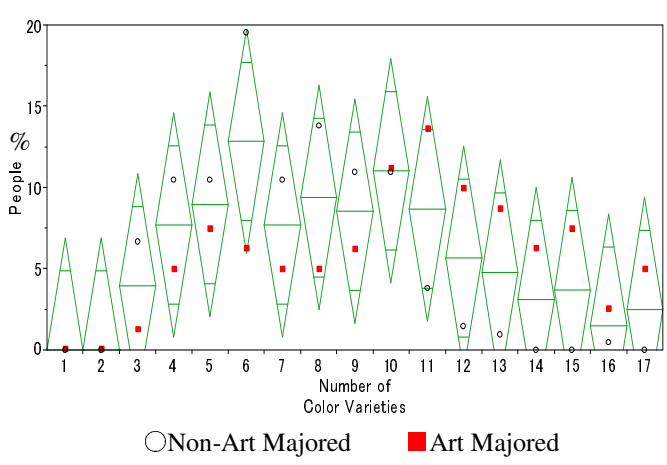

Figure 7. Number of color varieties based on major with means diamond in all categories

\section{3-7. Respondents' Parents' Backgrounds and Number of Color Varieties}

In this section, the respondents were asked if their parents have had any art or design background. Figure 8 shows the data for each region/category individually. It is observed that in all the regions, respondents whom had 
parents with art and/or design background had used more number of color varieties in their bedroom drawing in comparison with those who didn't. Figure 9 indicates the data as a whole and again, it is noted that respondents with parents with art background have used more number of color varieties in the drawing $(\mathrm{P}<0.05)$.
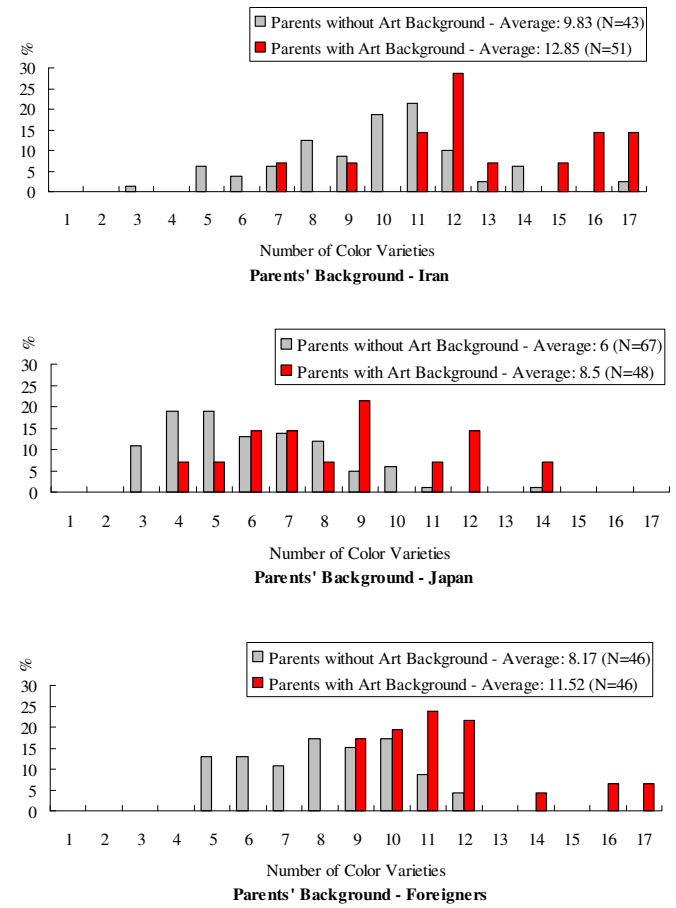

Figure 8. Number of color varieties and parents' background in each category

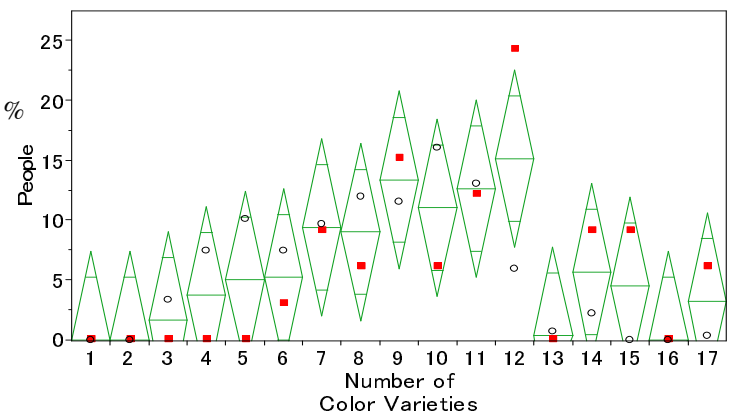

OParents with no art BG 口 Parents with art BG Figure 9. Number of color varieties and parents' background in the whole data

\section{3-8. Exterior and Interior Drawings}

This section discusses the number of color varieties used both in the interior (bedroom) drawing and the exterior drawing in all the regions (Figure 10). It is observed that in total, foreigners (including Iranians) used more number of color varieties compared to Japanese $(\mathrm{P}<0.05)$. Furthermore, Japanese subjects used more color varieties in the exterior drawing whereas Iranian and foreign respondents used more number of color varieties in the bedroom drawing rather than exterior $(\mathrm{P}<0.05)$.
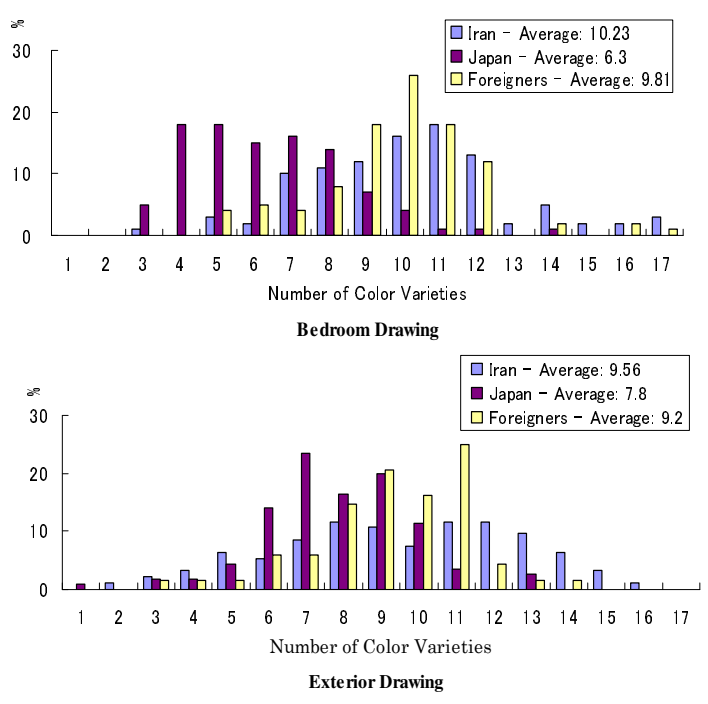

Figure 10. Number of color varieties in interior and exterior drawings

\section{3-9. Gender and Number of Color Varieties}

In this section, we discuss the number of color varieties used in the bedroom and exterior drawings by female and male subjects. Figure 11 shows the results of bedroom drawing for each category, whereas Figure 12 indicates the 
number of color varieties used in the exterior drawing. It is observed that foreign and Iranian female subjects used more number of color varieties in the bedroom drawing rather than the male subjects $(\mathrm{P}<0.05)$. However in Japan, male subjects used more number of color varieties in the bedroom drawing, although, this was not statistically significant. As for the exterior drawing, all the female subjects used more number of color varieties rather than the male subjects $(\mathrm{P}<0.05)$.
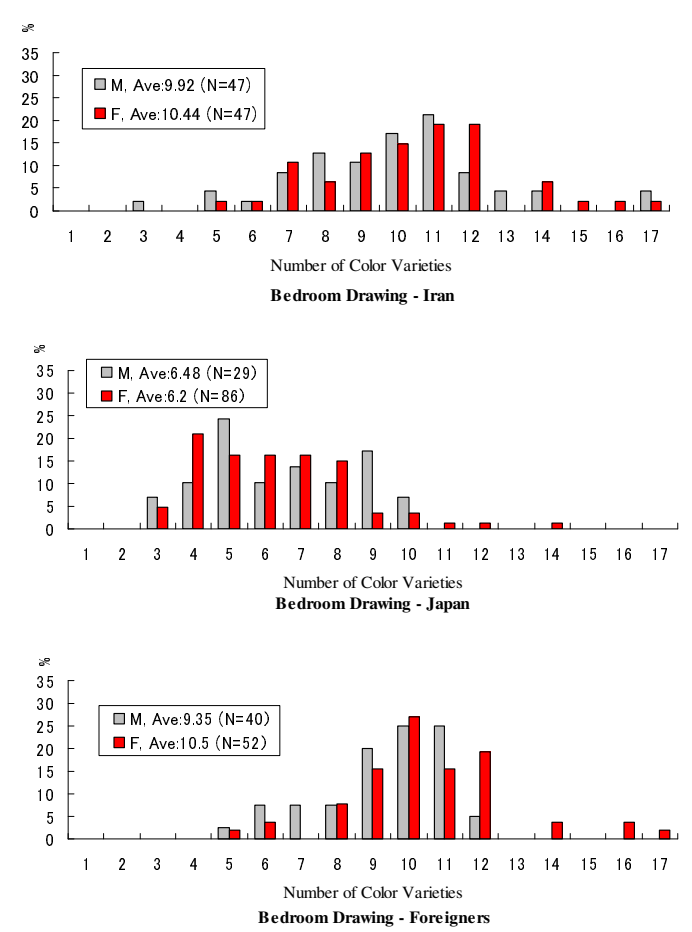

Figure 11. Number of color varieties in bedroom drawing and gender
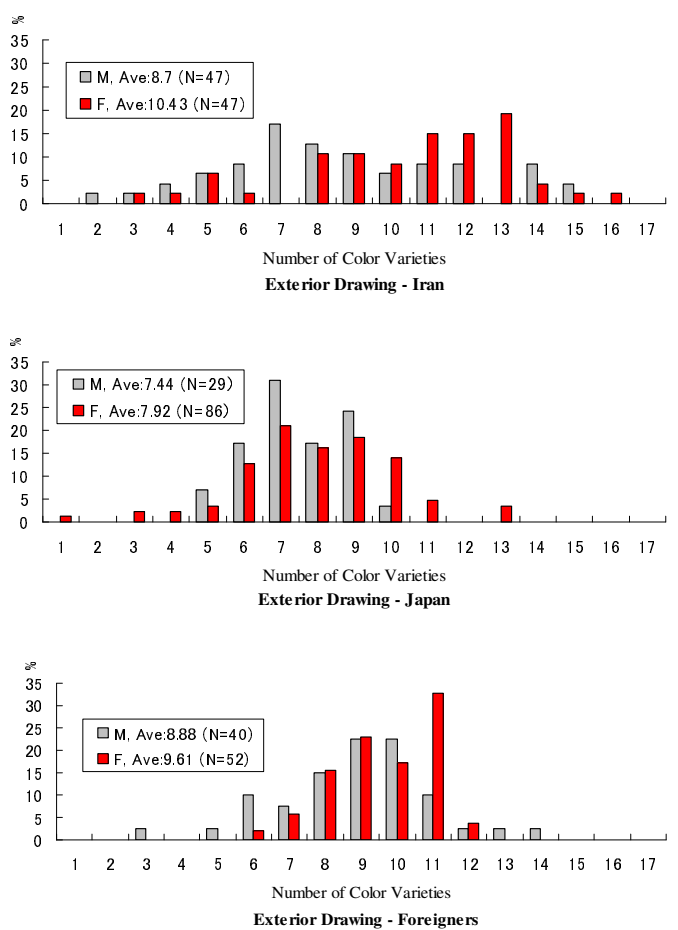

Figure 12. Number of color varieties in exterior drawing and gender

\section{3-10. Patterns used in the walls}

The reason for choosing drawings with color pencils was to see if the respondents will get creative in the drawings and use different patterns. Table 5 indicates that Iranians have used more patterns compared to the Japanese and foreign subjects $(\mathrm{P}<0.05)$. These patterns include: Striped, Mixed Shades, Flourish, and

Table 5. Patterns used in the Bedroom Wall Drawing

${ }^{*} \mathrm{p}<.05 ; * * \mathrm{p}<.01$

\begin{tabular}{|c|c|c|c|c|c|c|c|c|}
\hline & Plain & Patterned ${ }^{*}$ & Striped & Mixed Shades ${ }^{* *}$ & Flourish & Starish & Curved Lines & Circled \\
\hline $\begin{array}{l}\text { Iran } \\
(\mathrm{N}=94)\end{array}$ & 77 (81.9\%) & $17(18.1 \%)$ & $1(1.1 \%)$ & $9(9.6 \%)$ & $3(3.2 \%)$ & 0 & $2(2.1 \%)$ & $2(2.1 \%)$ \\
\hline $\begin{array}{l}\text { Japan } \\
(\mathrm{N}=115)\end{array}$ & $108(93.9 \%)$ & $7(6.1 \%)$ & 0 & $5(4.3 \%)$ & $1(0.9 \%)$ & 0 & $1(0.9 \%)$ & 0 \\
\hline $\begin{array}{l}\text { Foreigners } \\
(\mathrm{N}=92)\end{array}$ & $85(92.4 \%)$ & $7(7.6 \%)$ & 0 & 0 & $3(3.3 \%)$ & $2(2.2 \%)$ & 0 & $2(2.2 \%)$ \\
\hline
\end{tabular}


patterns using Curved Lines and Circles. Foreigners have used Starish Patterns too.

\section{3-11. Overall Results}

In this study, social environment was our primary focus and the influences of a person's residential, regional, educational and personal backgrounds were examined. It was observed that with more hours of art education, subjects used more number of color varieties. Therefore, it is indicated that among the case studies, education has a strong positive correlation with number of color varieties $(\mathrm{P}<0.01)$.

\section{Discussion and Conclusions}

In this paper, the influence of personal background on color preference was studied using a questionnaire and 2 drawings: bedroom drawing and exterior drawing. Since this was a cross cultural study, therefore, we tried to have the most international exterior drawing. Although, it can be assumed that with using another drawing, we could have got different results; however, since we observed the same results in all the categories (Iranians, Japanese and Foreigners) for all the case studies, therefore, it can be argued that we would have gotten the same results using another drawing too.

It was concluded that in total, more than $96.77 \%$ have used their favorite colors in the drawings. The favorite colors that were seen in all the categories (Iranians, Foreigners, and Japanese) included: Black, Green, Light Blue,
Blue, Purple, Pink, Light Pink, Red, Orange and Yellow, while Blue was the only color used in all the elements of the Drawings. Grieve ${ }^{29}$ and Maden et al. ${ }^{30}$ argue that blue is the color most frequently chosen by adults. Ward ${ }^{31}$ discusses that blue, red and green are the most popular colors. Similarly, Eysenck ${ }^{32}$ concluded that blue is the most preferred color, followed by red, green and purple.

There was not much difference seen between the city types in regional section regarding number of color varieties, although, people who have been living in Coastal areas have used less number of color varieties compared to subjects living in metropolitan or mountainous areas. Looking at the results, it was observed that subjects living in coastal areas have used more blue hues in the drawings $(\mathrm{P}<0.05)$. George M. Michaels ${ }^{33}$ argues that environment and social status have something to do with the development of preference for colors.

It was identified that education had a strong positive correlation with the number of color varieties $(\mathrm{P}<0.01)$. Furthermore, respondents whom were art and design majored had used more color varieties in their drawings $(\mathrm{P}<0.05)$. In addition, respondents whom had parents with art background had used more number of color varieties in their drawings compared to those who didn't $(\mathrm{P}<0.05)$. Crozier ${ }^{34}$ argued that the differences in color preference are due to the fact that the human reactions to color are conditioned by learned experiences. It has been demonstrated that the evaluative responses to products are affected not only by professional 
training (design vs. non-design), but also by duration of the training ( $1^{\text {st }}$ to $3^{\text {rd }}$ year design students and lecturers in design) ${ }^{35}$. Moreover, Li-Chen $\mathrm{Ou}$ et al. ${ }^{36}$ did a cross cultural comparison of color emotion among 223 observers from Britain, Taiwan, France, Germany, Spain, Sweden, Argentina and Iran, and found that effects of professional background (design vs. non-design) and age were strong on the like/dislike response.

Additionally, it was observed that Iranian and foreign respondents have used more number of color varieties in the drawings compared to the Japanese respondents $(\mathrm{P}<0.05)$. Moreover, Iranians and foreigners used more varieties in the interior rather than the exterior drawing, while the contrary was seen for the Japanese subjects $(\mathrm{P}<0.05)$. Furthermore, Iranians had used more patterns in the bedroom wall drawings including striped, mixed shapes, flourish, curved lined, and circled $(\mathrm{P}<0.05)$.

Although, gender was not the main focus of this research, but the correlation between gender and number of color varieties was examined. It was observed that Iranian and foreign female subjects used more number of color varieties in the interior drawing compared to the male subjects $(\mathrm{P}<0.05)$. However, in the exterior drawing, all the female subjects used more number of color varieties rather than the male subjects. It can be said this is because female subjects paid more attention to the details compared to the male subjects.

Although, this study and its method has not been done before, but comparing some of the results with other studies confirm the reliability of these results. However, we tried to take the color-preference-researches further and clearly identified the correlations between personal background and color preference. We hope that these data can be used as a base for other studies.

\section{Further Studies}

This study was only done in Iran and Japan. However, as for the influence of education, the same results were seen among foreigners, Iranians and Japanese subjects. Therefore, it is assumed that the same results will be seen in other countries as well. But having the chance, we would like to do this study, in other countries too. Moreover, we would like to have more focus on influences of art education on color preference. Additionally, the results can also be analyzed in terms of color emotion.

\section{Acknowledgments}

We are indebted to Professor Carole Faucher, Professor Takashi Hasumi and Professor Takashi Uzawa for kindly helping us in gathering the data.

This research was approved by the Research Ethics Committee of Faculty of Art and Design, University of Tsukuba before gathering the data. 


\section{References}

1) Mahshid Baniani and Hiroya Igarashi: How Different People with Different Life-Styles Think towards Interior Design, In AIC Color 2009, Sydney, pp. 124-125

2) Miho Saito: Preference for White in Japan and its Background - "A Comparative Study in Asian Areas", Journal of the Color Science Association of Japan, 1999, 23(3), pp. 158-337

3) Per K Gunnerod: Marketing Cut Flowers in Japan and Hong Kong, International Trade Forum, (July-September) 1991, 27, pp. 28-29

4) Taki Kanda: Analysis of Human Feelings to Colors, Knowledge-Based Intelligent Information and Engineering Systems, Lecture Notes in Computer Science, 2004, 3215, pp. 143-150

5) T. R. Garth: The color preference of five hundred fifty-nine full blood Indians, Journal of Experimental Psychology, 1922; 5, pp. 392-418

6) Laurence Jacobs, Charles Keown, Reginald Worthley, and Kyung-II Ghymn: Cross Cultural Color Comparisons: Global Marketers Beware! International marketing Review, 1991, 8(3), pp. 21-30

7) N. Silver: Sex and Racial Differences in Color and Number Preferences, Perceptual and Motor Skills, Feb. 1988, 66, pp. 295-299

8) Sjoerd Wiegersma and G. Van Der Elst: Blue Phenomenon: Spontaneity or Preference, Perceptual and Motor Skills, 1988, 66(2), pp. 308-310

9) Shoyama S, Tochihara Y, Kim J: Japanese and Korean ideas about clothing colors for elderly people; Intercountry and intergenerational differences, Color Research and Application, 2003, 28, pp.139-150

10) Hulbert A. C., Ling Y: Biological Components of sex differences in color preference, Current Biology, 2007, 17, pp. 623-625

11) J. P. Guilford, \& Patricia C. Smith: A System of Color Preferences, The American Journal of Psychology, Dec. 1959, 72(4), pp. 487-502

12) Thomas R. Garth and Electa P. Porter: The Color Preference of 1032 Young Children, The American Journal of Psychology, Jul. 1934, 46(3), pp. 448-451

13) E. Walton, Ruth B. Guilford, and J. P. Guilford: Color Preferences of 1279 University Students, The American Journal of Psychology, Apr. 1933, 45(2), pp. 322-328

14) L. J. Harris: Two sexes in the mind: Perceptual and creative differences between women and men, Journal of Creative Behavior, 23, pp.14-25

15) Walsh L. M., Toma R. B., Tuveson R. V., \& Sondhi L.: Color Preference and food Choice among Children, Journal of Psychology, 1990, 124(6), pp. 645-653

16) Marilyn A. Read and Deborah Upington: Young Children's Color Preferences in the Interior Environment, Journal of Early Childhood Education, 2009, 36, pp. 491-496

17) Zemach I. K, \& Teller D. Y.: Infant color vision: Infants' spontaneous color preferences are well behaved, Vision Research, 2007, 47, pp. 1362-1367

18) Meerum Terwogt M, \& Hoeslma J B: Colors and emotions: Preferences and combinations, Journal of General Psychology, 2005, 122(1), pp. 261-263

19) Pitchford N. J., \& Mullen K. T.: The role of perception, language, and preference in the development acquisition of basic color terms, Journal of Experimental Child Psychology, 2005, 90, pp. 275-302

20) Richard S. Cimbalo, Karen L. Beck, and Donna S. Sendziak: Emotionally Toned Pictures and Color Selection for Children and College Students, The Journal of Genetic Psychology, 1984, 133(2), pp.303-304 
21) Francis M. Adams, and Charles E. Osgood: A Cross-Cultural Study of the Affective Meanings of Color, Journal of Cross-Cultural Psychology, 1973, 4(2), pp. 135-156

22) Irvin L. Child, Jens A. Hansen and Frederick W. Hornbeck: Age and Sex Differences in Children's Color preferences, Child Development, Mar. 1968, 39(1), pp. 237-247

23) Vasanti Jadva, Melissa Hines, and Susan Golombok: Infants' Preferences for Toys, Colors, and Shapes: Sex Differences and Similarities, Archives of Sexual Behaviors, 2010, 39, pp. 1261-1273

24) H. J. Eysenck: A Critical and Experimental Study of Color Preference, The American Journal of Psychology, Jul. 1941, 54(3), pp. 385-391

25) G. W. Granger: An Experimental Study of Color Preferences, The Journal of General Psychology, 1955, 52, pp. 3-20

26) I. C. McManus, A. L. Jones, \& J. Cottrell: The Aesthetics of Color, Perception, 1981, 10, pp. 651-666

27) Neperud R. W. \& Freeman K.: Bases of children's visual preferences and discrimination, Visual Arts Research, 1988, 14(1), pp. 83-88

28) C. Newton: A Development Study of Aesthetic Response using both Verbal and Nonverbal Measures, Visual Arts Research, 1989, 15(1), pp. $76-85$

29) K.W. Grieve: Traditional Beliefs and Color Perception, Perceptual and Motor Skills, 1991, 72(4), pp. 1319-1323

30) Thomas J. Maden, Kelly Hewett, and Martin S. Roth: Managing Images in Different Cultures: A Cross-National Study of Color Meanings and Preferences, Journal of International marketing, 2000, 8(4), pp. 90-107

31) Gary Ward: Colors and Employee Stress Reduction, Supervision, Feb. 1995, 56, pp. 3-5

32) H. J. Eysenck: A critical and experimental study of color preferences, American Journal of
Psychology, 1941, 21, pp. 35-49

33) George M. Michaels: Color Preference According to Age, The American Journal of Psychology, Jan. 1924, 35(1), pp. 79-87

34) W. R. Crozier: The psychology of color preference, Review of Progress in Coloration and Related Topics, 1996, 26, pp. 63-72

35) Whitfield A. \& Wiltshire J: Design training and aesthetic evaluation: An intergroup comparison, Journal of Environmental Psychology, 1982, 2, pp. 109-117

36) Li-Chen Ou, M.Ronnier Luo, Pei-Li Sun, Neng-Chung Hu, Hung-Shing Chen, Shing-Sheng Guan, Andree Woodcock, Jose Luis Caivano, Rafael Huertas, Alain Tremeau, Monica Billger, Hossein Izadan, and Klaus Richter: A Cross-Cultural Comparison of Color Emotion for Two-Color Combinations, Color Research and Application, Feb. 2012, 37(1), pp 23-43

Mahshid Baniani, University of Tsukuba, 1-1-1 Tennodai, Tsukuba, Ibaraki, 305-8574, Japan m_bani@hotmail.com 